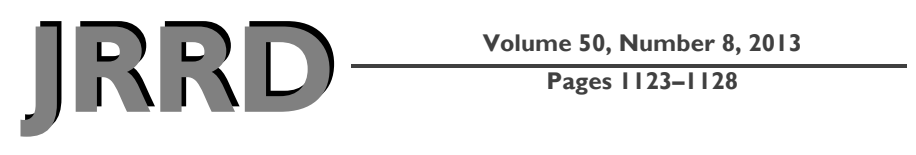

\title{
Functionality of i-LIMB and i-LIMB Pulse hands: Case report
}

\author{
Olga van der Niet; ${ }^{*}$ Raoul M. Bongers, MSc, PhD; ${ }^{2}$ Corry K. van der Sluis, MD, $\mathbf{P h D}^{\mathbf{1}}$ \\ ${ }^{1}$ University of Groningen, University Medical Center Groningen, Department of Rehabilitation Medicine, Center for \\ Rehabilitation Medicine, Groningen, the Netherlands; ${ }^{2}$ University of Groningen, University Medical Center Groningen, \\ Center of Human Movement Sciences, Groningen, the Netherlands
}

\begin{abstract}
The availability of various multiarticulated prosthetic hands makes determining differences in functionality between these hands relevant. The current study asked whether the functionality of these hands increased with time of use and whether grip force and robustness improved. A 45-year-old man with wrist disarticulation used the i-LIMB and the i-LIMB Pulse hands in a series of tests covering all functional levels as described in the framework of the International Classification of Functioning, Disability and Health. Using the i-LIMB for 1 yr improved Southampton Hand Assessment Procedure function scores. However, the i-LIMB Pulse did not improve much over 5 mo of training, possibly because of the intense training in the month prior to the first i-LIMB Pulse tests. The i-LIMB Pulse hand generally showed higher scores on the tests and better grip strength and robustness than the i-LIMB. The i-LIMB Pulse has overcome the shortcomings of the i-LIMB hand. The preset grip patterns simplified the complex control of the multiarticulated i-LIMB hand, which contributed to patient satisfaction.
\end{abstract}

Key words: activities of daily living, amputation, arm injuries, congenital abnormalities, multiarticulated, myoelectric, occupational therapy, prosthetics and implants, rehabilitation, upper extremity.

\section{INTRODUCTION}

The development of prosthetic hands seems to have gained renewed interest recently. In the last decade, research centers and manufacturers have been employing the current state of technology to develop new concepts and apply new materials and techniques in trying to cre- ate a more optimal replacement for a missing hand. A major step in these developments has been the introduction of myoelectrically controlled multiarticulated hands. These multiarticulated hands have the ability to fold around objects and perform various grip types, which make them more like a human hand than single-joint myoelectric prosthetic hands such as the Dynamic Mode Control (DMC) plus hand (Ottobock; Duderstadt, Germany). The first multiarticulated hand available on the market was the i-LIMB hand (2007, Touch Bionics; Livingston, United Kingdom) followed by the bebionic hand (2010, RSLSteeper; Leeds, United Kingdom), and the i-LIMB Pulse (2010, Touch Bionics).

Abbreviations: ACMC $=$ Assessment of Capacity for Myoelectric Control 2.0, DMC $=$ Dynamic Mode Control, IoF $=$ Index of Function, logits = log-odds, OPUS = Orthotics and Prosthetics Users' Survey, SHAP = Southampton Hand Assessment Procedure, T0 $=$ baseline measurements with DMC plus hand, $\mathrm{T} 1=$ measurements after $1 \mathrm{mo}$ of $\mathrm{i}$-LIMB usage, $\mathrm{T} 2=$ measurements after $1 \mathrm{yr}$ of $\mathrm{i}$-LIMB usage, T3 = measurements after 1 mo of i-LIMB Pulse usage, T4 = measurements after 5 mo of i-LIMB Pulse usage, TAPES $=$ Trinity Amputation and Prosthesis Experience Scales, VAS = visual analog scale.

*Address all correspondence to Olga van der Niet, Occupational Therapist, Department of Rehabilitation Medicine, Center for Rehabilitation Medicine, University Medical Center Groningen, PO Box 30.001, Groningen, $9700 \mathrm{RB}$, the Netherlands; +31-50-361-2295; fax: +31-50-361-9251.

Email: 0.van.der.niet@umcg.nl

http://dx.doi.org/10.1682/JRRD.2012.08.0140 
In an earlier study, we presented a case in which we compared the multiarticulated i-LIMB hand and the single-joint DMC plus hand [1]. Surprisingly, we found no clear functional advantage for the i-LIMB over the DMC plus hand. Compared with the DMC plus hand, the iLIMB hand had a higher reliability when holding objects but less strength and robustness. The functional scores of the i-LIMB were equal to or even lower than those of the DMC plus hand. However, the participant had used the DMC plus hand for 18 mo but trained with the i-LIMB hand for only $4 \mathrm{wk}$.

The current study is a follow-up to that earlier study and concentrates on two questions. First, Does the time period in which the multiarticulated i-LIMB hand is used affect its functional scores? Second, Is the i-LIMB Pulse hand an improved version of the i-LIMB hand, at least in strength and robustness? To answer these questions, a single participant with amputation used an i-LIMB hand and an i-LIMB Pulse hand for 5 mo.

\section{CASE DESCRIPTION}

In November 2006, a 43-year-old man sustained a wrist disarticulation on his dominant left side during work. Initially, he was provided with a two-electrode myoelectric prosthesis with a DMC plus hand and a passive wrist rotator.

In December 2008, through the efforts of his personal injury lawyer, the patient received a new prosthesis with an i-LIMB hand and a rigid wrist, and in December 2010, he was provided with a third prosthesis with an iLIMB Pulse hand and a friction wrist. All three of the patient's prostheses had forearm sockets with proximal trimlines inferior to the humeral condyles. Supination to $-10^{\circ}$ was possible with and without a prosthesis, and $45^{\circ}$ and $80^{\circ}$ of pronation were measured with and without a prosthesis, respectively.

\section{METHODS}

After obtaining written informed consent and approval of the local medical ethics review board, the patient performed a series of tests with the three prosthetic hands at different instances in time. The tests with the DMC plus hand and the i-LIMB hand after 1 mo of training (T1) were described previously [1].
In the current study, the i-LIMB was tested again after 1 yr of usage (T2) and the i-LIMB Pulse was tested after 1 mo of training (T3) and approximately 5 mo of training and daily use (T4). In addition to performing the same tests as in our previous study, we performed a qualitative interview with the patient.

The conducted tests covered all functional levels as described in the framework of the International Classification of Functioning, Disability and Health for Function scores and Activity and Participation scores [2].

\section{Function}

Grip and pinch strength were measured using the Jamar dynamometer (E-Link, Biometrics Ltd; Newport, United Kingdom) in five positions and the pinch meter as advocated by the American Society of Hand Therapists [3-4]. However, single-try measurements were applied.

Prehensile patterns and grip postures were assessed by the Southampton Hand Assessment Procedure (SHAP). The SHAP consists of 26 tasks: 12 abstract object tasks and 14 activities of daily living. The time necessary to complete each task was recorded and transformed into a functionality profile that reflected the ability to perform several grip types. Overall functionality is reflected in the Index of Function (IoF). Nondisabled participants score between 94 and 99 on a scale of 0-100 [5-6].

The Assessment of Capacity for Myoelectric Control 2.0 (ACMC) gauges myoelectric control in an everyday activity [6]. We examined the task "packing a suitcase." A score of zero log-odds (logits) is an average control ability [7].

\section{Activity and Participation}

Satisfaction with the prosthesis in general and the influence of the prosthesis on performing activities of daily living were measured by the Trinity Amputation and Prosthesis Experience Scales (TAPES) in four psychosocial subscales (score 14-70, higher scores reflect better adjustment), four activity-restriction subscales (score 0-12, higher scores reflect more restrictions), and a single prosthesis-satisfaction subscale (score 10-50, higher scores reflect higher satisfaction) [8].

The Orthotics and Prosthetics Users' Survey (OPUS) assessed function, satisfaction, and quality of life related to the patients' use of the upper-limb prosthesis. The Upper Extremity Functional Status is established from a 19-item daily activity questionnaire (score: $0-57$, Figure). 


\begin{tabular}{|c|c|c|c|c|c|c|c|}
\hline \multirow[t]{2}{*}{$\begin{array}{l}\text { Please Indicate How Easily You } \\
\text { Perform the Following Activities }\end{array}$} & \multicolumn{5}{|c|}{$\begin{aligned} 3 & =\text { Very Easy } \\
2 & =\text { Easy } \\
1 & =\text { Difficult } \\
0 & =\text { Not Able } \\
\text { NA } & =\text { Not Applicable }\end{aligned}$} & \multicolumn{2}{|c|}{$\begin{array}{l}\text { Do You Usually } \\
\text { Perform this } \\
\text { Activity Using } \\
\text { Your Prosthesis? }\end{array}$} \\
\hline & 3 & 2 & 1 & 0 & NA & Yes & No \\
\hline \multicolumn{8}{|l|}{ 1. Wash Face } \\
\hline \multicolumn{8}{|l|}{ 2. Put Toothpaste and Brush Teeth } \\
\hline \multicolumn{8}{|l|}{ 3. Brush/Comb Hair } \\
\hline \multicolumn{8}{|l|}{ 4. Put on and Remove T-Shirt } \\
\hline \multicolumn{8}{|l|}{ 5. Button Shirt with Front Buttons } \\
\hline \multicolumn{8}{|l|}{ 6. Attach End of Zipper and Zip Jacket } \\
\hline \multicolumn{8}{|l|}{ 7. Put on Socks } \\
\hline \multicolumn{8}{|l|}{ 8. Tie Shoe Laces } \\
\hline \multicolumn{8}{|l|}{ 9. Use Fork/Spoon } \\
\hline \multicolumn{8}{|l|}{ 10. Poor from $12 \mathrm{oz}$ Can } \\
\hline \multicolumn{8}{|l|}{ 11. Write Name Legibly } \\
\hline \multicolumn{8}{|l|}{ 12. Use Scissors } \\
\hline \multicolumn{8}{|l|}{ 13. Open Door with Knob } \\
\hline \multicolumn{8}{|l|}{ 14. Carry Laundry Basket } \\
\hline \multicolumn{8}{|l|}{ 15. Dial Touch Tone Telephone } \\
\hline \multicolumn{8}{|l|}{ 16. Fold Bath Towel } \\
\hline \multicolumn{8}{|l|}{ 17. Open Envelope } \\
\hline \multicolumn{8}{|l|}{ 18. Stir in Bowl } \\
\hline 19. Put on and Take off Prosthesis & & & & & & & \\
\hline
\end{tabular}

Figure.

Orthotics and Prosthetics Users' Survey Upper Extremity Functional Status questionnaire.

A score of 27 reflects zero logits and a moderate level of upper-limb function [9-10].

\section{Prosthesis Characteristics}

Visual analog scale (VAS) scores were used to determine the patient's subjective opinion on the strength, appearance, sound, precision grip, power grip, and robustness of the prosthetic hands (lowest score 0, highest score 10). The patient also scored the relevance of these prosthetic characteristics. A semistructured qualitative interview gave the patient the opportunity to express his experience with the tests and the outcomes.

\section{RESULTS}

\section{Function}

Table 1 presents the scores from the Jamar dynamometer, pinch meter, SHAP, and ACMC. Grip strength was highly variable. The power grip force of the i-LIMB after 1 yr was remarkably low in positions $1,2,3$, and 4
Table 1.

Function scores over time (T0-T4) for i-LIMB and i-LIMB Pulse hands: grip strength and pinch strength measured with Jamar dynamometer and pinch meter, respectively; Southampton Hand Assessment Procedure (SHAP); SHAP Index of Function (IoF) scores; and log-odds scores of Assessment of Capacity for Myoelectric Control 2.0 (ACMC).

\begin{tabular}{|c|c|c|c|c|c|}
\hline \multirow{2}{*}{ Measure } & \multirow{2}{*}{$\begin{array}{c}\text { DCM } \\
\text { Plus } \\
\text { T0* }^{*}\end{array}$} & \multicolumn{2}{|c|}{ i-LIMB } & \multicolumn{2}{|c|}{ i-LIMB Pulse } \\
\hline & & $\mathbf{T} 1^{*}$ & T2 & T3 & $\mathbf{T 4}$ \\
\hline \multicolumn{6}{|c|}{ Grip Strength (N) } \\
\hline 1 & 76 & 14 & 1 & 13 & 11 \\
\hline 2 & 88 & 13 & 3 & 26 & 44 \\
\hline 3 & 91 & 65 & 31 & 106 & 97 \\
\hline 4 & 96 & 57 & 50 & 105 & 81 \\
\hline 5 & 36 & 20 & 43 & 107 & 85 \\
\hline \multicolumn{6}{|c|}{ Pinch Strength (N) } \\
\hline Lateral & NA & 16 & 10 & 20 & 17 \\
\hline Tripod & 121 & 15 & 16 & 26 & 21 \\
\hline Tip & NA & 20 & 12 & 19 & 17 \\
\hline \multicolumn{6}{|l|}{ SHAP } \\
\hline Spherical & 90 & 90 & 90 & 94 & 94 \\
\hline Power & 75 & 51 & 85 & 88 & 90 \\
\hline Tip & 39 & 42 & 58 & 74 & 51 \\
\hline Tripod & 76 & 32 & 84 & 87 & 88 \\
\hline Lateral & 69 & 23 & 62 & 80 & 81 \\
\hline Extension & 81 & 55 & 45 & 87 & 84 \\
\hline IoF & 74 & 52 & 76 & 88 & 87 \\
\hline
\end{tabular}

*DMC plus and i-LIMB scores as revealed in our earlier study [1].

DMC = Dynamic Mode Control, NA = not applicable, $\mathrm{T} 0=$ baseline measurements with DMC plus hand, T1 = measurements after 1 mo of i-LIMB usage, T2 = measurements after $1 \mathrm{yr}$ of $\mathrm{i}$-LIMB usage, $\mathrm{T} 3$ = measurements after $1 \mathrm{mo}$ of i-LIMB Pulse usage, T4 = measurements after 5 mo of i-LIMB Pulse usage.

of the Jamar dynamometer. Power grip and pinch grip were higher for the i-LIMB Pulse than the i-LIMB.

With regard to the SHAP, the IoF score after $1 \mathrm{yr}$ of $\mathrm{i}$ LIMB use was substantially higher than the IoF score after 1 mo of i-LIMB use. This change over time was less for the i-LIMB Pulse. Importantly, the scores on the SHAP were generally higher for the i-LIMB Pulse. The tip grip score for the i-LIMB Pulse was lower after 5 mo than after 1 mo.

Myoelectric control, as reflected by ACMC scores, was most favorable in the i-LIMB Pulse. The ACMC score was lowest for the i-LIMB after $1 \mathrm{yr}$, but still well above the average of zero logits. 


\section{Activity and Participation}

In the TAPES (Table 2), the differences in scores over time were small for both hands. The psychosocial adjustment score and the prosthesis satisfaction score were somewhat higher for the i-LIMB Pulse than for the i-LIMB, whereas for the activity-restriction score, it was the other way around.

Performance of daily activities (functional status) in the OPUS decreased over time for the i-LIMB, while the i-LIMB Pulse scores increased over time.

\section{Prosthesis Characteristics}

VAS scores in Table 3 revealed that, in general, both prostheses were rated high. The i-LIMB Pulse was rated higher on produced force and robustness of the hand, and those were relevant prosthetic properties for this user.

Table 2.

Activity and participation scores measured by Trinity Amputation and Prosthesis Experience Scales (TAPES) and Orthotics and Prosthetics Users' Survey (OPUS) for different measurements over time (T0-T4).

\begin{tabular}{|c|c|c|c|c|c|}
\hline \multirow{2}{*}{ Measure } & \multirow{2}{*}{$\begin{array}{c}\text { DCM } \\
\text { Plus } \\
\text { T0 }^{*}\end{array}$} & \multicolumn{2}{|c|}{ i-LIMB } & \multicolumn{2}{|c|}{ i-LIMB Pulse } \\
\hline & & T1* & $\mathbf{T} 2$ & T3 & T4 \\
\hline
\end{tabular}

\section{TAPES}

Psychosocial Adjustment

$\begin{array}{lrrrrr}\text { General Adjustment } & 12 & 11 & 11 & 11 & 12 \\ \text { Social Adjustment } & 20 & 20 & 20 & 20 & 20 \\ \text { Adjustment to Limitation } & 5 & 9 & 9 & 15 & 11 \\ \text { Optimal Adjustment } & 10 & 10 & 9 & 9 & 10 \\ \text { Total } & 47 & 50 & 49 & 55 & 53\end{array}$

Activity Restriction

Athletic Restriction

Social Restriction

Occupational Restriction

Total

Prosthesis Satisfaction

$\begin{array}{lrrrrr}\text { Aesthetic Satisfaction } & 13 & 18 & 16 & 16 & 17 \\ \text { Weight Satisfaction } & 3 & 3 & 4 & 4 & 4 \\ \text { Functional Satisfaction } & 18 & 19 & 18 & 20 & 21 \\ \text { Total } & 34 & 40 & 38 & 40 & 42 \\ \text { OPUS Functional Status } & 30 & 29 & 25 & 23 & 30\end{array}$

${ }^{*}$ DMC plus and i-LIMB scores as revealed in our earlier study [1]. ACMC = Assessment of Capacity for Myoelectric Control 2.0, DMC = Dynamic Mode Control, OPUS = Orthotic and Prosthetics Users' Survey, SHAP $=$ Southampton Hand Assessment Procedure, T0 = baseline measurements with DMC plus hand, T1 = measurements after 1 mo of i-LIMB usage, $\mathrm{T} 2=$ measurements after $1 \mathrm{yr}$ of i-LIMB usage, T3 = measurements after $1 \mathrm{mo}$ of i-LIMB Pulse usage, T4 = measurements after 5 mo of i-LIMB Pulse usage.
The patient especially liked the ease of grasping with the multiarticulated hand. He said that the hand had more grip, because of the roughness of the glove (robotic skin). Furthermore, with the multiarticulated hand, the positioning of the fingers around an object did not need to be as precise as with hands with single-degree-of-freedom finger joints (e.g., DMC plus hand). Moreover, the possibility of being able to perform a tip grip with this multiarticulated hand added a lot of functionality. The patient reported that he highly valued the larger force of the i-LIMB Pulse over the i-LIMB, because it made objects feel more secure in the hand. The preset grip patterns of the i-LIMB Pulse were judged as a considerable advantage, mainly because changing between hand positions took less time and was more reliable than with the iLIMB hand.

\section{DISCUSSION}

We saw a major improvement in the use of the iLIMB hand over time, as reflected in the SHAP scores. This improvement seemed to be mainly due to learning to exploit the possibilities that the multiple grip patterns of the hand offered compared with a single-joint prosthetic hand. This application of various grip patterns is what the patient had to learn when he first received a multiarticulated hand. Presumably, when he received the i-LIMB Pulse, he was familiar with the possibilities that a multiarticulated hand offered. Hence, we did not find a learning effect for the i-LIMB Pulse on the SHAP scores. The friction wrists of the DMC plus hand and the i-LIMB Pulse were used in full supination to perform the lateral objects and zipper tasks of the SHAP (at T0 [baseline measurements with DMC plus hand] and T3). At T4, the patient performed the same tasks in pronation with a pure lateral grip, because he was more able to preposition this hand.

The effects of training are not easy to extract from a single case study, but they are probably quite important. When the patient received his first multiarticulated hand, he received limited training because the therapist was not familiar with the device. After he received the i-LIMB Pulse, all novel possibilities were thoroughly explored to tailor the features to the patient's individual needs. The patient received intensive, daily training during the first month after delivery of the i-LIMB Pulse, which probably contributed to the high SHAP scores after 1 mo of training and the stable scores over time. 
Table 3.

Visual analog scale (VAS) scores (0-10): patients' judgments regarding characteristics (Char) of prosthetic hands and relevancy (Rel) of these characteristics.

\begin{tabular}{|c|c|c|c|c|c|c|c|c|c|c|}
\hline \multirow[t]{2}{*}{ VAS } & \multicolumn{2}{|c|}{$\begin{array}{c}\text { DMC Plus } \\
\left(\mathrm{TO}^{*}\right)\end{array}$} & \multicolumn{2}{|c|}{ i-LIMB (T1*) } & \multicolumn{2}{|c|}{ i-LIMB (T2) } & \multicolumn{2}{|c|}{$\begin{array}{l}\text { i-LIMB Pulse } \\
\text { (T3) }\end{array}$} & \multicolumn{2}{|c|}{$\begin{array}{l}\text { i-LIMB Pulse } \\
\text { (T4) }\end{array}$} \\
\hline & Char & Rel & Char & Rel & Char & Rel & Char & Rel & Char & Rel \\
\hline Power (none-much) & 10 & 10 & 6 & 10 & 7 & 8 & 8 & 8 & 9 & 8 \\
\hline Look (ugly-beautiful) & 5 & 5 & 9 & 5 & 10 & 4 & 8 & 5 & 9 & 5 \\
\hline Sound (noisy-quiet) & 5 & 5 & 6 & 5 & 5 & 4 & 7 & 6 & 3 & 5 \\
\hline Tripod Grip (bad-good) & 5 & 8 & 8 & 8 & 8 & 8 & 8 & 8 & 9 & 9 \\
\hline Reliability Holding Objects (low-high) & 7 & 10 & 10 & 10 & 8 & 9 & 9 & 8 & 9 & 9 \\
\hline Robustness (vulnerable-solid) & 9 & 10 & 6 & 10 & 6 & 9 & 8 & 8 & 8 & 9 \\
\hline
\end{tabular}

*DMC plus and i-LIMB scores as revealed in our earlier study [1].

DMC = Dynamic Mode Control, T0 = baseline measurements with DMC plus hand, $\mathrm{T} 1=$ measurements after 1 mo of i-LIMB usage, T2 = measurements after 1 yr of i-LIMB usage, T3 = measurements after 1 mo of i-LIMB Pulse usage, T4 = measurements after 5 mo of i-LIMB Pulse usage.

After 1 yr of i-LIMB use (T2), we did not find improvements in patient satisfaction, adjustment to limitations, or ease in execution of daily tasks, as reflected in TAPES and OPUS scores. Scores had even decreased slightly. We believe that the initial scores with the i-LIMB (T1) were relatively high because the patient put a lot of effort into obtaining the i-LIMB, which may have colored his judgment. We think that the scores became more realistic over the year of use. A further issue to consider is whether technical decline of the i-LIMB contributed to these results. Service maintenance was performed on the iLIMB hand 3 mo before the remeasurements at $\mathrm{T} 2$. Although service maintenance just before remeasurements should be preferred, the patient did not complain about any functional deficits of the i-LIMB. As such, we do not expect that technical degradation substantially influenced our results. The increase of OPUS scores with the i-LIMB Pulse stemmed from a real improvement in use of the prosthesis, which was also reflected in the positive remarks that the user made during the conducting of the tests.

Strength, robustness, and the preset grip patterns were clear improvements of the i-LIMB Pulse over the iLIMB hand, although the dynamometry scores were highly variable, which was probably due to variations in the way the handle was grasped over trials. A better indication of grip strength might have been achieved by averaging several measurements at each grip position of the dynamometer. Furthermore, execution of daily tasks was quicker with the i-LIMB Pulse. Additional innovations, such as the preset grip patterns, were highly appreciated and may be of great value to prosthesis users.
As such, we conclude that the second generation of multiarticulated hands has gained functionality over the first generation. The differences between the hands were not substantial, but this was to be expected given that the design of the two hands was very much alike.

If we compare the functionality of the i-LIMB Pulse to the DMC plus hand [1], the power grip of the i-LIMB Pulse equals the power grip of the DMC plus hand, which was of great value to the prosthesis user. The pinch grip of the i-LIMB Pulse has improved compared with the i-LIMB, but is clearly lower than that of the DMC plus hand. However, to avoid crushing delicate objects, a modest pinch grip may be preferred over a powerful pinch grip. Patient-rated satisfaction (TAPES), ease of execution of daily tasks (OPUS), and myoelectric control (ACMC) scores were more favorable for the i-LIMB Pulse than the DMC plus hand. The i-LIMB Pulse scored higher on the ACMC than the DMC plus hand because, when using the i-LIMB Pulse, the patient showed better adjustment of grip force, adjustment of the opening width of the hand, timing, coordination with both hands, and handling of delicate objects.

\section{CONCLUSIONS}

Within the limitations of a single case study, we conclude that the functionality of the i-LIMB hand improved after 1 yr of usage and is comparable with the DMC plus hand. This patient, who was an experienced singledegree-of-freedom hand user, needed exploration, training, and experience to achieve high functionality with the 
multiarticulated prosthetic hand. We also conclude that the i-LIMB Pulse has overcome the i-LIMB hand's shortcomings in terms of strength and robustness. The preset grip patterns simplified the complex control of the multiarticulated i-LIMB hand, which also contributed to patient satisfaction. Although multiarticulated prosthetic hands still cannot replace sound hands, their development seems to be a large step toward this ultimate goal.

\section{ACKNOWLEDGMENTS}

\author{
Author Contributions: \\ Study concept and design: C. K. van der Sluis, R. M. Bongers. \\ Acquisition of data: O. van der Niet. \\ Analysis and interpretation of data: O. van der Niet, R. M. Bongers, \\ C. K. van der Sluis. \\ Drafting of manuscript: O. van der Niet. \\ Critical revision of manuscript for important intellectual content: \\ R. M. Bongers, C. K. van der Sluis. \\ Study supervisor: C. K. van der Sluis.
}

Financial Disclosures: The authors have declared that no competing interests exist.

Funding/Support: This material was unfunded at the time of manuscript preparation.

Institutional Review: After obtaining written informed consent and agreement of the local medical ethics review board, the patient performed a series of tests with the three prosthetic hands at different instances in time.

Participant Follow-Up: Participants have been informed of the publication of this study.

\section{REFERENCES}

1. van der Niet OV, Reinders-Messelink HA, Bongers RM, Bouwsema H, Van Der Sluis CK. The i-LIMB hand and the DMC plus hand compared: A case report. Prosthet Orthot Int. 2010;34(2):216-20. [PMID:20470060] http://dx.doi.org/10.3109/03093641003767207

2. World Health Organization. International Classification of Functioning, Disability and Health. Geneva (Switzerland): World Health Organization; 2001.

3. Innes E. Handgrip strength testing: A review of the literature. Aust Occup Ther J. 1999;46:120-40. http://dx.doi.org/10.1046/j.1440-1630.1999.00182.x

4. MacDermid JC, Evenhuis W, Louzon M. Inter-instrument reliability of pinch strength scores. J Hand Ther. 2001; 14(1):36-42. [PMID:11243558] http://dx.doi.org/10.1016/S0894-1130(01)80023-5
5. Light CM, Chappell PH, Kyberd PJ. Establishing a standardized clinical assessment tool of pathologic and prosthetic hand function: Normative data, reliability, and validity. Arch Phys Med Rehabil. 2002;83(6):776-83.

[PMID:12048655]

http://dx.doi.org/10.1053/apmr.2002.32737

6. Metcalf CD, Woodward H, Wright V, Chappell PH, Burridge $\mathrm{JH}$, Yule VT. Changes in hand function with age and normative unimpaired scores when measured with the Southampton Hand Assessment Procedure. Hand Ther. 2008;13(3):79-83.

7. Hermansson LM, Fisher AG, Bernspång B, Eliasson AC. Assessment of capacity for myoelectric control: A new Rasch-built measure of prosthetic hand control. J Rehabil Med. 2005;37(3):166-71. [PMID:16040474]

8. Desmond DM, MacLachlan M. Factor structure of the Trinity Amputation and Prosthesis Experience Scales (TAPES) with individuals with acquired upper limb amputations. Am J Phys Med Rehabil. 2005;84(7):506-13. [PMID:15973087] http://dx.doi.org/10.1097/01.phm.0000166885.16180.63

9. Heinemann AW, Bode RK, O'Reilly C. Development and measurement properties of the Orthotics and Prosthetics Users' Survey (OPUS): A comprehensive set of clinical outcome instruments. Prosthet Orthot Int. 2003;27(3):191-206. [PMID:14727700] http://dx.doi.org/10.1080/03093640308726682

10. Burger H, Franchignoni F, Heinemann AW, Kotnik S, Giordano A. Validation of the Orthotics and Prosthetics User Survey Upper Extremity Functional Status module in people with unilateral upper limb amputation. J Rehabil Med. 2008;40(5):393-99. [PMID:18461266] http://dx.doi.org/10.2340/16501977-0183

Submitted for publication August 4, 2012. Accepted in revised form January 24, 2013.

This article and any supplementary material should be cited as follows:

van der Niet O, Bongers RM, van der Sluis CK. Functionality of i-LIMB and i-LIMB Pulse hands: Case report. J Rehabil Res Dev. 2013;50(8):1123-28. http://dx.doi.org/10.1682/JRRD.2012.08.0140

ResearcherID/ORCID: Corry K. van der Sluis, MD, PhD: C-1102-2012; Raoul M. Bongers, MSc, PhD: C-1094-2012

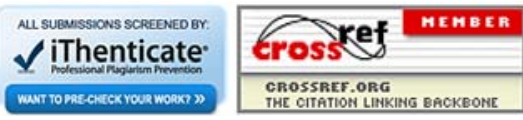

